\title{
Predictive factors of 30-day mortality in patients with traumatic subdural hematoma
}

\author{
IULIA-SEVASTIANA PASTOR ${ }^{1}$, LĂCRIMIOARA PERJU DUMBRAVĂ ${ }^{2}, \operatorname{COSTEL~SISERMAN~}^{3}$, \\ HORAȚIU STAN $^{1}$, IOANA PARA ${ }^{4}$ and IOAN ȘTEFAN FLORIAN ${ }^{1}$
}

\author{
${ }^{1}$ Department of Neurosurgery, ${ }^{2}$ First Department of Neurology, and Departments of ${ }^{3}$ Legal Medicine and ${ }^{4}$ Internal Medicine, \\ Faculty of Medicine, 'Iuliu Hațieganu' University of Medicine and Pharmacy, 400012 Cluj-Napoca, Romania
}

Received March 19, 2021; Accepted April 19, 2021

DOI: $10.3892 /$ etm.2021.10189

\begin{abstract}
In the present study, we aimed to assess and analyze the predictive factors of 30-day mortality in patients with acute subdural hematoma (ASDH) who underwent surgical intervention after traumatic brain injury (TBI). We conducted a retrospective study, which included a cohort of 135 consecutive patients diagnosed with ASDH who required surgical evacuation. We assessed the demographic and clinical data, the imaging data of the hematoma described by preoperative computed tomography (CT) and the type of neurosurgical intervention for hematoma evacuation via either craniectomy or craniotomy. The patients were followed up for 30 days after head trauma and the occurrence of death was noted. Death was recorded in $63(46.6 \%)$ patients at 30 days after TBI. There was a significant number of deceased patients who underwent craniectomy (71.4\%). The Glasgow Coma Scale (GCS) was statistically significantly lower in patients who died $(\mathrm{P}<0.001)$, with a cut-off value of $\leq 12$, under which the probability of death increased [AUC 0.830 (95\% CI, 0.756-0.889); Se 90.48\% (95\% CI, 80.4-96.4); Sp 66.7\% (95\% CI, 54.6-77.3); P<0.001]. The midline shift was statistically significantly higher in deceased patients $(\mathrm{P}=0.005)$, with a cut-off value of $>7 \mathrm{~mm}$, over which the probability of death increased [AUC 0.637 (95\% CI, 0.550-0.718); Se 38.1\% (95\% CI, 26.1-51.2); Sp 86.1\% (95\% CI, 75.9-93.1); $\mathrm{P}=0.003$. There were significantly more deceased patients with intracranial hypertension, brain herniation, brain swelling, intraparenchymal hematoma and cranial fracture. In multivariate analysis only a Glasgow score $\leq 12$ and a midline shift $>7 \mathrm{~mm}$ were independently linked to mortality. Brain herniation and intraparenchymal hematoma were associated
\end{abstract}

Correspondence to: Dr Ioana Para, Department of Internal Medicine, Faculty of Medicine, 'Iuliu Haţieganu' University of Medicine and Pharmacy, 8 Victor Babes Street, 400012 Cluj-Napoca, Romania

E-mail: ioana.para@yahoo.com

Key words: acute subdural hematoma, traumatic brain injury, neurosurgery, predictive factors, mortality with a higher probability of dying, but the statistical threshold was slightly exceeded. The type of neurosurgery performed for patients with ASDH was not an independent predictive factor for 30-day mortality. However, craniectomy was associated with a higher mortality in patients with ASDH.

\section{Introduction}

Subdural hemorrhage often occurs in traumatic brain injury (TBI) conditions and in some cases tends to have unfavorable clinical outcomes. By far, neurosurgery is still the most important lifesaving procedure. As other studies have shown, the neurosurgical management of TBI is challenging and still requires more evidence $(1,2)$. Some researchers explain that the outcome of patients operated on for acute subdural hematoma (ASDH) is dependent on their preoperative status. Moreover, they studied some predictive factors of mortality in patients with ASDH after TBI. For a long time, clinical data showed a significant correlation between old age and poor outcome (3). Recently, studies have found other key factors involved in patient outcome. Status on admission, evaluated by the Glasgow Coma Scale (GCS), is one factor which is correlated with patient mortality (4). Initial computed tomography (CT) findings such as midline shift, hematoma thickness, and brain swelling were also found to be predictive factors for postoperative status $(4,5)$. The context of polytrauma and the mechanism of injuries are definitely significant for the status of patients and for achieving independence of living at discharge (6). Traffic accidents are the cause of the highest mortality rates in patients due to head trauma (7). Finally, the mortality rate is high in patients with associated pathologies (7).

Furthermore, researchers have discussed the best neurosurgical procedure for the treatment of ASDH. Neurosurgical guidelines contain surgical indications for the evacuation of ASDH based on the size of the hematoma and the midline shift. It is clear that patients with ASDH who present intracranial pressure or neurological dysfunction require emergent surgical decompression (1,8-10). However, it is still unclear whether the bone flap should be removed (craniectomy) or replaced (craniotomy) when an extracranial herniation is expected to develop after surgery (8). Also researchers have investigated whether the surgical intervention is independently correlated with the mortality rate, but the results are inconclusive (11-13). 
Table I. Demographic and clinical data of the patients with ASDH.

\begin{tabular}{lccr}
\hline Variables & Deceased $(\mathrm{n}=63)$ & Survivors (n=72) & P-value \\
\hline Age (years) & $71(59 ; 80)$ & $71(64 ; 77.7)$ & 0.600 \\
Sex & & & \\
$\quad$ Female & $17(27 \%)$ & $20(27.8 \%)$ & $>0.999$ \\
Male & $46(73 \%)$ & $52(72.2 \%)$ & \\
Operation & & & \\
$\quad$ Craniectomy & $45(71.4 \%)$ & $33(45.8 \%)$ & \\
Craniotomy & $18(28.6 \%)$ & $39(54.2 \%)$ & $<0.001$ \\
GCS & $4(3 ; 8)$ & $14(8 ; 15)$ & $<0.001$ \\
GCS, $\mathrm{n}(\%)$ & & & \\
$>12$ & $6(9.5 \%)$ & $48(66.7 \%)$ & 0.146 \\
$\leq 12$ & $57(90.5 \%)$ & $24(33.3 \%)$ & 0.644 \\
Atrial fibrillation and atrial flutter, $\mathrm{n}(\%)$ & $18(28.6 \%)$ & $12(16.7 \%)$ & 0.952 \\
Arterial hypertension, $\mathrm{n}(\%)$ & $22(34.9 \%)$ & $29(40.3 \%)$ & 0.410 \\
Epilepsy, $\mathrm{n}(\%)$ & $5(7.9 \%)$ & $7(9.7 \%)$ & 0.941 \\
Type 2 diabetes mellitus, $\mathrm{n}(\%)$ & $9(14.3 \%)$ & $14(19.3 \%)$ & \\
Chronic ethanolism, $\mathrm{n}(\%)$ & $11(17.5 \%)$ & & \\
\hline
\end{tabular}

ASDH, acute subdural hematoma; GCS, Glasgow Coma Scale.

In this study, we aimed to assess and analyze the predictive factors of 30-day mortality in patients with ASDH undergoing surgery after TBI.

\section{Patients and methods}

The nature of this study was retrospective, longitudinal, analytical, and observational. The study included a cohort of 135 consecutive patients with ASDH admitted to the Department of Neurosurgery of the Emergency County Hospital in Cluj-Napoca, between January 2018 and December 2019. Either the patients or their families (where applicable) signed an informed consent form for study inclusion. This study was approved by the Clinical Ethics Committee of the 'Iuliu Haţieganu' University of Medicine and Pharmacy in Cluj-Napoca, Romania.

Patients diagnosed with ASDH after TBI who required surgical evacuation (craniectomy or craniotomy) were eligible for inclusion in this study. We excluded patients who suffered from cardiorespiratory arrest before surgery (successfully resuscitated or otherwise), patients with terminal diseases, patients with a recent history (the last 30 days) of acute coronary syndrome, decompensated heart failure, upper gastrointestinal bleeding or major surgery.

We assessed the following parameters: The demographic characteristics of each patient, comorbidities [atrial fibrillation and atrial flutter, arterial hypertension, epilepsy, type 2 diabetes mellitus, alcohol use disorder (AUD)], mechanisms of head injury, admission status of TBI according to the GCS, radiographic characteristics of the hematoma on preoperative $\mathrm{CT}$, and surgical procedure (craniectomy or craniotomy). Patients were followed up for 30 days after surgery, and the occurrence of death was noted. The mechanism of head injury was classified as follows: Fall from the same level, fall from another level, traffic accidents, or an unknown mechanism.
The following imaging data were recorded: Thickness of the subdural hematoma, midline shift, presence of brain swelling, presence of brain herniation, signs of intracranial hypertension. Other intracranial lesions caused by TBI were also logged: Cranial fracture, extradural hematoma, subdural hemorrhage, intraparenchymal hematoma, contusion and laceration.

The same preoperative management, intensive care unit, anesthesia protocols and consent forms were applied for each patient. Patients underwent one type of neurosurgical intervention: Hematoma evacuation via either craniectomy or craniotomy. For craniotomy, the bone flap was placed back and secured with multiple marginal sutures once the hematoma was evacuated and rigorous hemostasis was achieved, in the event that brain swelling was not significant. If considerable brain edema or signs of brain herniation and severely increased intracranial pressure occurred, the flap was typically larger and removed entirely.

Statistical analysis was carried out using the MedCalc Statistical Software version 19.4.1 (MedCalc Software Ltd., Ostend, Belgium; https://www.medcalc.org; 2020). Quantitative data were tested for normality of distribution using the Shapiro Wilk test and are characterized by median and 25, 75 percentiles. Qualitative data are expressed as frequency and percentage. Comparisons between groups were performed using the Mann-Whitney or the Chi-square test, whenever appropriate. Multivariate logistic regression was used to evaluate the independent association between data and mortality. Variables that achieved statistical significance in univariate analysis were introduced in the predictive model. A P-value $<0.05$ was considered statistically significant.

\section{Results}

Death was recorded in $63(46.6 \%)$ patients at 30 days after TBI. Clinical and demographic data can be found in Table I. 
Table II. Imaging data of the patients with ASDH.

\begin{tabular}{|c|c|c|c|}
\hline Variables & Deceased $(n=63)$ & Survivors $(n=72)$ & P-value \\
\hline Intracranial hypertension, n (\%) & $52(82.5 \%)$ & $38(52.8 \%)$ & 0.001 \\
\hline Thickness of subdural hematoma (mm) & $21(14 ; 28)$ & $18(13 ; 26)$ & 0.100 \\
\hline \multicolumn{4}{|l|}{ Localization of subdural hematoma, n (\%) } \\
\hline Right & $30(47.6 \%)$ & $26(36.1 \%)$ & \\
\hline Left & $28(44.4 \%)$ & $34(47.2 \%)$ & \\
\hline Bilateral & $5(7.9 \%)$ & $12(16.7 \%)$ & \\
\hline Midline shift (mm) & $6(15 ; 11)$ & $4(0 ; 6)$ & 0.005 \\
\hline \multicolumn{4}{|l|}{ Midline shift >7, n (\%) } \\
\hline$\leq 7$ & $39(61.9 \%)$ & $62(86.1 \%)$ & 0.002 \\
\hline$>7$ & $24(38.1 \%)$ & $10(13.9 \%)$ & \\
\hline Brain herniation, $\mathrm{n}(\%)$ & $23(36.5 \%)$ & $5(6.9 \%)$ & $<0.001$ \\
\hline Brain swelling, n (\%) & $51 \quad(81 \%)$ & $38(52.8 \%)$ & 0.001 \\
\hline Signs of meningeal irritation, $\mathrm{n}(\%)$ & $11(17.5 \%)$ & $7(9.7 \%)$ & 0.287 \\
\hline \multicolumn{4}{|l|}{ Mechanism of injury, $\mathrm{n}(\%)$} \\
\hline Unknown conditions & $12(19.0 \%)$ & $7 \quad(9.7 \%)$ & 0.159 \\
\hline Falls at the other level & $12(19.0 \%)$ & $10(13.9 \%)$ & \\
\hline Falls at the same level & $34(54.0 \%)$ & $52(72.2 \%)$ & \\
\hline Traffic accidents & $5(7.9 \%)$ & $3(4.2 \%)$ & \\
\hline Extradural hematoma, n (\%) & $9(14.3 \%)$ & $6(8.3 \%)$ & 0.410 \\
\hline Cranial fracture, n (\%) & $25(39.7 \%)$ & $11(15.3 \%)$ & 0.001 \\
\hline Intraparenchymal hematoma, n (\%) & $21(33.3 \%)$ & $10(13.9 \%)$ & 0.013 \\
\hline \multicolumn{4}{|l|}{ Contusion and laceration, $\mathrm{n}(\%)$} \\
\hline No & $38(60.3 \%)$ & $57(79.2 \%)$ & 0.055 \\
\hline Contusion & $6(9.5 \%)$ & $3(4.2 \%)$ & \\
\hline Contusion and dilaceration & $19(30.2 \%)$ & $19(30.2 \%)$ & \\
\hline
\end{tabular}

ASDH, acute subdural hematoma.

Most of the patients who died underwent craniectomy (71.4\%). GCS was statistically significantly lower in patients that died $(\mathrm{P}<0.001)$. For GCS, we found a cut-off value of $\leq 12$, under which the probability of death increased [AUC 0.830 (95\% CI, 0.756-0.889); Se 90.48\% (95\% CI, 80.4-96.4); Sp 66.7\% (95\% CI, 54.6-77.3); P<0.001].

The comparison between the deceased patients and survivors, regarding the imaging data, is summarized in Table II. There were significantly more deceased patients compared with survivors with intracranial hypertension [52 (82.5\%) vs. 38 (52.8\%); $\mathrm{P}=0.001$ ], brain herniation [23 (36.5\%) vs. 5 (6.9\%); $\mathrm{P}<0.001$, brain swelling [51 (81\%) vs. 38 (52.8\%); $\mathrm{P}=0.001]$, intraparenchymal hematoma $[21(33.3 \%)$ vs. $10(13.9 \%)$, $\mathrm{P}=0.013]$, and cranial fracture $[25(39.7 \%)$ vs. $11(15.3 \%)$; $\mathrm{P}=0.001]$. For the midline shift, we found a cut-off value of $>7 \mathrm{~mm}$, over which the probability of death increased [AUC0.637 (95\% CI, 0.550-0.718); Se 38.1\% (95\% CI, 26.1-51.2); Sp 86.1\% (95\% CI, 75.9-93.1), $\mathrm{P}=0.003$ ].

Multivariate analysis was performed in order to determine which variables were independently associated with death at 30 days after the head trauma (Table III). Variables that achieved statistical significance in the univariate analysis were introduced in the predictive model. Only a Glasgow score $\leq 12$ and a midline shift $>7 \mathrm{~mm}$ were independently linked to mortality. Brain herniation and intraparenchymal hematoma were associated with a higher probability of death, but the statistical threshold was slightly exceeded.

\section{Discussion}

ASDH is a clinical entity occurring in different contexts of head trauma. Compared to the past, the mortality rate of ASDH has decreased and is currently around 14\% (14). Despite the developments in neurosurgery and the urgent intervention, ASDH still has an unfavorable clinical outcome (15). The literature data show that the outcome of ASDH after surgery is dependent on multiple factors (16). In this study, we also aimed to obtain an accurate early prediction for the clinical outcome of ASDH in the context of head trauma.

We performed a statistical analysis of the supposed factors for 30-day mortality in patients who underwent surgery for ASDH after TBI. In the univariate analysis, we compared the number of deaths to that of survivors. Death was recorded in $63(46.6 \%)$ patients at 30 days after the head trauma. In the analysis of clinical and demographic factors, we found a statistically significant result for the Glasgow Coma Scale (GCS), which was significantly lower in patients who died at 30 days after head trauma $(\mathrm{P}<0.001)$. In our study, the probability of 
Table III. Multivariate analysis of the 30-day mortality.

$95 \% \mathrm{CI}$, for OR

\begin{tabular}{|c|c|c|c|c|c|}
\hline & \multirow[b]{2}{*}{$\mathrm{B}$} & \multirow[b]{2}{*}{ P-value } & \multirow[b]{2}{*}{ OR } & \\
\hline & & & & Lower & Upper \\
\hline Craniotomy & 0.581 & 0.424 & 1.788 & 0.430 & 7.428 \\
\hline $\mathrm{GCS} \leq 12$ & 2.515 & $<0.001$ & 12.369 & 3.915 & 39.079 \\
\hline Intracranial hypertension & 0.340 & 0.567 & 1.405 & 0.439 & 4.496 \\
\hline Midline shift $>7 \mathrm{~mm}$ & 1.259 & 0.037 & 3.521 & 1.079 & 11.489 \\
\hline Brain herniation & 1.133 & 0.071 & 3.104 & 0.907 & 10.625 \\
\hline Brain swelling & 0.644 & 0.416 & 1.903 & 0.404 & 8.958 \\
\hline Cranial fracture & 0.480 & 0.372 & 1.616 & 0.563 & 4.637 \\
\hline Intraparenchimal hematoma & 0.970 & 0.085 & 2.639 & 0.875 & 7.963 \\
\hline Constant & 0.371 & 0.416 & 1.449 & & \\
\hline
\end{tabular}

OR, odds ratio; CI, confidence interval; GCS, Glasgow Coma Scale.

death increased under a cut-off value $\leq 12$ for GCS $(\mathrm{P}<0.001)$. In the analysis of imaging data, we found that patients who developed secondary brain injuries such as intracranial hypertension, brain herniation, brain swelling had a significant risk of dying at 30 days after TBI. Patients who presented associated lesions such as intraparenchymal hematoma and cranial fracture on admission also had a significantly high risk of death. In our study, the midline shift was statistically significantly associated with death and had a cut-off value of $>7 \mathrm{~mm}$, over which the probability of death increased $(\mathrm{P}=0.003)$.

We performed a multivariate analysis of the variables that were statistically significant in univariate analysis. We wanted to ascertain which variable was an independent predictive factor for 30-day mortality. In our analysis, GCS was independently correlated with mortality. GCS showed the clinical severity on admission for our patients. In a wide range of hospitals, this clinical scale is useful for assessing the level of consciousness and coma (17). Lavrador et al found in their study that a GCS $\leq 8$ on admission was statistically significantly correlated with a worse outcome $(\mathrm{P}<0.05)(6)$. Prognosis of ASDH, particularly in the elderly, was evaluated in a systematic review that included seven eligible studies. The researchers investigated the predictive factors and modified index and they found that an initial GCS of 3-8 was the most important predictive factor for a negative outcome (18). In a retrospective study focusing on the criteria for surgery in elderly patients with ASDH, a GCS of 3-8 was a significant indicator for high mortality $(\mathrm{P}<0.001)(19)$.

The second independent factor in our analysis was the midline shift with a cut-off value of $>7 \mathrm{~mm}$. As we observed in other studies, the presence and size of the midline shift was also an important determinant of the outcome in this study (20). In a retrospective analysis with 67 patients, all over 70 years of age, a midline shift $>20 \mathrm{~mm}$ was an independent parameter for 10-day mortality after surgery, with a median survival rate of 3.5 days (range 2-7) (21). In the same retrospective study quoted by us (19), the researchers compared the outcome, evaluated by the Glasgow Outcome Scale (GOS) at discharge and the midline shift. There was a statistically significant difference between the worst outcome mean GOS 1 (death), midline shift $>10 \mathrm{~mm}$ and good recovery mean GOS 5, midline shift $1.8 \mathrm{~mm}$ (range: 0-10 $\mathrm{mm}$ ) (19).

Multivariate analysis showed that brain herniation and intraparenchymal hematoma were associated with a higher probability of death as the P-values (brain herniation $\mathrm{P}=0.071$ and intraparenchymal hematoma $\mathrm{P}=0.085$ ) were close to the statistically significant threshold. Probably, a higher number of patients introduced in our analysis would yield the expected results.

Discussing the type of surgery that the patients underwent, in our study we observed that craniectomy was more frequent than craniotomy. Craniectomy showed a higher association with mortality, but we could not prove its prediction for death. There is a worldwide large variation regarding the initial neurosurgical management of ASDH (22). Kolias et al reported in their analysis that a higher proportion of neurosurgeons from European countries $(48 / 110 ; 44 \%)$ performed craniectomy in more than half of ASDH cases $(\mathrm{P}<0,001)$ compared to UK/Irish neurosurgeons $(29 / 138 ; 21 \%)$ (23). Some studies did not report any therapeutic advantage of craniectomy vs. craniotomy (24). Jehan et al showed that primary craniectomy is not superior in outcome to craniotomy for the evacuation of intracranial hemorrhage after TBI (25).

Regarding the limitations of the study, we consider that a higher number of patients included in the study would provide significant results for the variables, close to the statistically significant threshold.

In conclusion, $\mathrm{ASDH}$ is a severe intracranial lesion in a high percentage of cases. In our study, we confirmed that some clinical and imaging data are predictive factors for mortality at 30 days after TBI. The following data were statistically significant: GCS with a cut-off value of $\leq 12$; secondary brain injuries such as intracranial hypertension, brain herniation, brain swelling; associated cranial lesions such as intraparenchymal hematoma, cranial fracture and midline shift with a cut-off value $>7 \mathrm{~mm}$. Moreover, in multivariate analysis, GCS $\leq 12$ and midline shift $>7 \mathrm{~mm}$ were independent predictive factors for 30-day mortality after head trauma. The type of neurosurgery had no statistically significant result regarding mortality. However, we observed that craniectomy was associated with a higher mortality in patients with ASDH. 


\section{Acknowledgements}

Not applicable.

\section{Funding}

No funding was received.

\section{Availability of data and materials}

The generated and analyzed data are included in this published article.

\section{Authors' contributions}

Conceptualization of the study was accomplished by ISP and IȘF. Methodology was the responsibility of ISP, LPD, CS, HS, IP and IŞF. Validation of the findings were undertaken by LPD, CS, HS, IP and ISTF. Formal analysis and investigation was the responsibility of ISP. Resources were acquired by ISP and IȘF. Writing-original draft preparation was done by ISP. Writing-review and editing was performed by ISP. Visualization of the concept and development of the study was the responsibility of ISP, LPD, CS, HS, IP and ISTF carried out the study supervision. All authors read and approved the final version of the manuscript.

\section{Ethics approval and consent to participate}

Either the patients or their families (where applicable) signed an informed consent form for study inclusion. This study was approved by the Clinical Ethics Committee of the 'Iuliu Hațieganu' University of Medicine and Pharmacy in Cluj-Napoca, Romania (nr. 307/28.09.2020).

\section{Patient consent for publication}

Not applicable.

\section{Competing interests}

The authors declare that they have no competing interests.

\section{References}

1. Van Essen TA, Den Boogert HF, Cnossen MC, de Ruiter GCW, Haitsma I, Polinder S, Steyerberg EW, Menon D, Maas AIR, Lingsma HF, et al: Variation in neurosurgical management of traumatic brain injury: A survey in 68 centers participating in the CENTER-TBI study. Acta Neurochir (Wien) 161: 435-449, 2019.

2. Phan K, Moore JM, Griessenauer C, Dmytriw AA, Scherman DB, Sheik-Ali S, Adeeb N, Ogilvy CS, Thomas A and Rosenfeld JV: Craniotomy versus decompressive craniectomy for acute subdural hematoma: Systematic review and meta-analysis. World Neurosurg 101: 677-685, 2017.

3. Howard MA III, Gross AS, Dacey RG Jr and Winn HR: Acute subdural hematomas: An age-dependent clinical entity. J Neurosurg 71: 858-863, 1989

4. Massaro F, Lanotte M, Faccani G and Triolo C: One hundred and twenty-seven cases of acute subdural haematoma operated on: Correlation between CT scan findings and outcome. Acta Neurochir (Wien) 138: 185-191, 1996.

5. Baucher G, Troude L, Pauly V, Bernard F, Zieleskiewicz L and Roche PH: Predictive factors of poor prognosis after surgical management of traumatic acute subdural hematomas: A single-center series. World Neurosurg 126: 944-952, 2019.
6. Lavrador JP, Teixeira JC, Oliveira E, Simão D, Santos MM and Simas N: Acute subdural hematoma evacuation: Predictive factors of outcome. Asian J Neurosurg 13: 565-571, 2018.

7. Alagoz F, Yildirim AE, Sahinoglu M, Korkmaz M, Secer M, Celik H, Yel C, Guvenc Y, Uckun OM, Narin F, et al: Traumatic acute subdural hematomas: Analysis of outcomes and predictive factors at a single center. Turk Neurosurg 27: 187-191, 2017.

8. Rush B, Rousseau J, Sekhon MS and Griesdale DE: Craniotomy versus craniectomy for acute traumatic subdural hematoma in the united states: A national retrospective cohort analysis. World Neurosurg 88: 25-31, 2016.

9. Carney N, Totten AM, O'Reilly C, Ullman JS, Hawryluk GW, Bell MJ, Bratton SL, Chesnut R, Harris OA, Kissoon N, et al: Guidelines for the management of severe traumatic brain injury, fourth edition. Neurosurgery 80: 6-15, 2017.

10. Haddad S, Aldawood AS, Alferayan A, Russell NA, Tamim HM and Arabi YM: Relationship between intracranial pressure monitoring and outcomes in severe traumatic brain injury patients. Anaesth Intensive Care 39: 1043-1050, 2011.

11. Gerard C and Busl KM: Treatment of acute subdural hematoma. Curr Treat Options Neurol 16: 275, 2014.

12. Hatashita S, Koga N, Hosaka Y and Takagi S: Acute subdural hematoma: Severity of injury, surgical intervention, and mortality. Neurol Med Chir (Tokyo) 33: 13-18, 1993.

13. Kolias AG, Scotton WJ, Belli A, King AT, Brennan PM, Bulters DO, Eljamel MS, Wilson MH, Papadopoulos MC, Mendelow AD, et al: Surgical management of acute subdural haematomas: Current practice patterns in the United Kingdom and the Republic of Ireland. Br J Neurosurg 27: 330-333, 2013.

14. Ryan CG, Thompson RE, Temkin NR, Crane PK, Ellenbogen RG and Elmore JG: Acute traumatic subdural hematoma: Current mortality and functional outcomes in adult patients at a level I trauma center. J Trauma Acute Care Surg 73: 1348-1354, 2012.

15. Godlewski B, Pawelczyk A, Pawelczyk T, Ceranowicz K, Wojdyn $\mathrm{M}$ and Radek M: Retrospective analysis of operative treatment of a series of 100 patients with subdural hematoma. Neurol Med Chir (Tokyo) 53: 26-33, 2013.

16. Won YD, Na MK, Ryu JI, Cheong JH, Kim JM, Kim CH and Han MH: Radiologic factors predicting deterioration of mental status in patients with acute traumatic subdural hematoma. World Neurosurg 111: e120-e134, 2018.

17. Teasdale G and Jennett B: Assessment of coma and impaired consciousness. A practical scale. Lancet 2: 81-84, 1974.

18. Evans LR, Jones J, Lee HQ, Gantner D, Jaison A, Matthew J, Fitzgerald MC, Rosenfeld JV, Hunn MK and Tee JW: Prognosis of acute subdural hematoma in the elderly: A systematic review. J Neurotrauma 36: 517-522, 2019.

19. Petridis AK, Dörner L, Doukas A, Eifrig S, Barth H and Mehdorn M: Acute subdural hematoma in the elderly; clinical and CT factors influencing the surgical treatment decision. Cent Eur Neurosurg 70: 73-78, 2009.

20. D'Amato L, Piazza O, Alliata L, Sabia G, Zito G, Frassanito L, Della Corte F and Tufano R: Prognosis of isolated acute post-traumatic subdural haematoma. J Neurosurg Sci 51: 107-111, 2007.

21. Benedetto N, Gambacciani C, Montemurro N, Morganti R and Perrini P: Surgical management of acute subdural haematomas in elderly: Report of a single center experience. Br J Neurosurg 31: 244-248, 2017

22. Van Essen TA, Volovici V, Cnossen MC, Kolias A, Ceyisakar I, Nieboer D, Peppel LD, Heijenbrok-Kal M, Ribbers G, Menon D, et al: Comparative effectiveness of surgery in traumatic acute subdural and intracerebral haematoma: Study protocol for a prospective observational study within CENTER-TBI and Net-QuRe. BMJ Open 9: 033513, 2019.

23. Kolias AG, Belli A, Li LM, Timofeev I, Corteen EA, Santarius T, Menon DK, Pickard JD, Kirkpatrick PJ and Hutchinson PJ: Primary decompressive craniectomy for acute subdural haematomas: Results of an international survey. Acta Neurochir (Wien) 154: 1563-1565, 2012

24. Woertgen C, Rothoerl RD, Schebesch KM and Albert R: Comparison of craniotomy and craniectomy in patients with acute subdural haematoma. J Clin Neurosci 13: 718-721, 2006.

25. Jehan F, Azim A, Rhee P, Khan M, Gries L, O'Keeffe T, Kulvatunyou N, Tang A and Joseph B: Decompressive craniectomy versus craniotomy only for intracranial hemorrhage evacuation: A propensity matched study. J Trauma Acute Care Surg 83: 1148-1153, 2017. 